University of Texas at El Paso

ScholarWorks@UTEP

3-1-2021

\title{
How Much For a Set: General Case of Decision Making Under Set- Valued Uncertainty
}

\author{
Laxman Bokati \\ The University of Texas at El Paso, Ibokati@utep.edu \\ Olga Kosheleva \\ The University of Texas at El Paso, olgak@utep.edu \\ Vladik Kreinovich \\ The University of Texas at El Paso, vladik@utep.edu
}

Follow this and additional works at: https://scholarworks.utep.edu/cs_techrep

Part of the Computer Sciences Commons

Comments:

Technical Report: UTEP-CS-21-05b

Published in Julia Rayz, Victor Raskin, Scott Dick, and Vladik Kreinovich (eds.), Explainable Al

and Other Applications of Fuzzy Techniques, Proceedings of the Annual Conference of the North American Fuzzy Information Processing Society NAFIPS'2021, West Lafayette, Indiana, June 7-9, 2021, Springer, Cham, Switzerland, 2022, pp. 52-61.

\section{Recommended Citation \\ Bokati, Laxman; Kosheleva, Olga; and Kreinovich, Vladik, "How Much For a Set: General Case of Decision Making Under Set-Valued Uncertainty" (2021). Departmental Technical Reports (CS). 1538. \\ https://scholarworks.utep.edu/cs_techrep/1538}

This Article is brought to you for free and open access by the Computer Science at ScholarWorks@UTEP. It has been accepted for inclusion in Departmental Technical Reports (CS) by an authorized administrator of ScholarWorks@UTEP.For more information, please contact Iweber@utep.edu. 


\title{
How Much For a Set: General Case of Decision Making Under Set-Valued Uncertainty
}

\author{
Laxman Bokati, Olga Kosheleva, and Vladik Kreinovich
}

\begin{abstract}
In real life, we often need to make a decision, i.e., we need to select one of the possible alternatives. In many practical situations, our objective is financial: we need to select an alternative that will bring us the largest financial gain. The problem is that usually, we only know the gain corresponding to each alternative with some uncertainty: instead of the exact numerical value of this gain, there is a whole set of possible values of this gain. How can we make decisions under such interval-valued uncertainty? An answer to this question is known for the case when these sets are closed. In this paper, we extend this result of the general case, when sets are not necessarily closed.
\end{abstract}

\section{Decision Making Under Set-Valued Uncertainty: Formulation of the Problem}

We need to make decisions. In real life, all the time, we need to make decisions. In many cases, decisions are made based on financial consequences:

- we want to select an investment strategy that will lead us to the largest gain,

- the company wants to select the design of a new plant so as to get the largest possible profit after it becomes operational, etc.

Decision making: ideal case. In the ideal case, we know the exact consequences of each possible alternative. In this case, decision is easy: we select the alternative for which the expected gain is the largest.

Decision making: realistic case. In practice, we rarely know the exact consequences of each possible alternative: this happens, e.g., when we invest the money into the

Laxman Bokati, Olga Kosheleva, and Vladik Kreinovich

University of Texas at El Paso, 500 W. University, El Paso, TX 79968, USA

e-mail: lbokati@miners.utep.edu, olgak@utep.edu, vladik@utep.edu 
government bonds, which is usually not the most profitable investment. In general, we only know these consequences with some uncertainty. In other words, for each alternative, instead of a single monetary value of the corresponding financial consequences, we have a whole set of possible values.

This set $S$ is usually bounded:

- there is an upper bound on possible gains, and

- there is an upper bound on possible losses - which means there is a lower bound on possible gains.

We need to select an alternative based on such bounded sets.

Decision making under set-valued uncertainty reduces to providing numerical estimates for the sets. The simplest situation is when we have only two alternatives:

- for one of them we know the exact monetary value $v$, and

- for the other one, we have a set $S$ of possible values.

In this case, we need to decide which of these two alternatives is better. A practical example can be deciding:

- whether to invest in government bonds - for which the future monetary value is well known,

- or to invest in a more volatile financial instrument such as stocks.

Here:

- If the value $v$ is very small, then clearly the set-valued alternative is better: we will denote this preference by $v<S$.

- When the value $v$ is very large, then clearly this numerical value is better: $S<v$.

One can see that there exists a threshold value $v(S)$-the supremum of all the values $v$ for which $v<S$ and the infimum of all the values $v$ for which $S<v-$ such that:

- for all $v<v(S)$, we have $v<S$, and

- for all $v>v(S)$, we have $S<v$.

So, for any positive number $\varepsilon>0$, no matter how small it is, we have

$$
v(S)-\varepsilon<S<v(S)+\varepsilon .
$$

When the value $\varepsilon$ is sufficiently small, the users cannot distinguish between the values $v(S)-\varepsilon, v(S)$, and $v(S)+\varepsilon$. In this sense, the set $S$ is equivalent to the numerical value $v(S)$; we will denote this by $S \equiv v(S)$.

This way, for each set, we get an equivalent numerical value - a "price" for this set. Once we know such prices, it is easy to make a decision in the general case of decision making under set-valued uncertainty: if we have alternatives described by sets $S_{1}, \ldots, S_{n}$ then,

- since each set $S_{i}$ is equivalent to its price $v\left(S_{i}\right)$,

- we simply select the alternative $i$ with the largest possible price $v\left(S_{i}\right)$. 
So, to be able to make decisions under set-values uncertainty, we need to be able to assign a price $v(S)$ to each bounded set.

What we do in this paper. In this paper, we provide an answer to this question. For this, first, in Section 2, we recall what is known: what is known is how to describe the price of closed sets. Then, in Section 3, we explain why the set of possible gains is not always closed, and we show that ideas - similar to the ones described in Section 2 - can help to describe the price of all possible bounded sets - not necessarily closed ones.

\section{What Is Known: Cases of Closed Intervals and Closed Sets}

Reasonable properties of the desired price function $v(S)$. Let us use common sense to come up with reasonable properties of the desired price function $v(S)$.

First, if some number $r$ is smaller than the greatest lower bound inf $S$ of the set $S$, this implies that this number is smaller than all numbers $s \in S$. Here, the set $S$ is the set of all possible gains. So the above condition means that the gain $r$ is worse than all possible gains $s \in S$. Thus, $r$ is worse than the whole set-valued alternative $S$ :

$$
r<S .
$$

In view of our price-based description of preference between the alternatives, the condition $r<S$ is equivalent to $r<v(S)$. Therefore, if $r<\inf (S)$, then we have $r<v(S)$. In particular, for any $\varepsilon>0$, we have inf $S-\varepsilon<\inf S$, thus we have inf $S-\varepsilon<v(S)$. In the limit $\varepsilon \rightarrow 0$, this implies that inf $S \leq v(S)$.

Similarly, if some number $r$ is larger than the least upper bound $\sup S$ of the set $S$, this implies that this number is larger than all numbers $s \in S$. Here, the set $S$ is the set of all possible gains. So the above condition means that the gain $r$ is better than all possible gains $s \in S$. Therefore, $r$ is better than the whole set-valued alternative $S$ : $S<r$. In view of our price-based description of preference between the alternatives, the condition $S<r$ is equivalent to $v(S)<r$. Thus, if $\sup (S)<r$, then we have $v(S)<r$. In particular, for any $\varepsilon>0$, we have $\sup S<\sup S+\varepsilon$, hence we have $v(S)<\sup S+\varepsilon<v(S)$. In the limit $\varepsilon \rightarrow 0$, this implies that $v(S) \leq \sup S$.

So, in general, we have

$$
\inf S \leq v(S) \leq \sup S .
$$

Another property comes from the fact that usually, we make several different decisions. Suppose that we make two consequent independent decisions, and let us consider two alternatives corresponding to these two decisions. Let us denote the set of possible gains from the first alternative by $S_{1}$, and the set of all possible gains from the second alternative by $S_{2}$. Then, by definition of the price function, for us the first alternative is equivalent to the numerical value $v\left(S_{1}\right)$, and the second alternative is 
equivalent to the numerical value $v\left(S_{2}\right)$. Thus, overall, for these two alternatives, we are willing to pay the price $v\left(S_{1}\right)+v\left(S_{2}\right)$.

We can view this situation differently: as a single complex alternative in which the set of possible gains is the set of all possible sums $s_{1}+s_{2}$, where $s_{1} \in S_{1}$ and $s_{2} \in S_{2}$. The set of all such sums is known as the Minkowksi sum of the two sets, it is denoted by $S_{1}+S_{2}$ :

$$
S_{1}+S_{2} \stackrel{\text { def }}{=}\left\{s_{1}+s_{2}: s_{1} \in S_{1} \& s_{2} \in S_{2}\right\} .
$$

For this set $S_{1}+S_{2}$, by definition of the price function, we pay the price

$$
v\left(S_{1}+S_{2}\right)
$$

However, this is the exact same situation as before, when we estimated the overall price of this situation as $v\left(S_{1}\right)+v\left(S_{2}\right)$. So, these two estimates must coincide, i.e., we must have

$$
v\left(S_{1}+S_{2}\right)=v\left(S_{1}\right)+v\left(S_{2}\right) .
$$

What is a closed set: reminder. A set $S$ is called closed if for every sequence of elements $s_{n} \in S$ from this set that converges to some value $s$, the limit point $s$ also belongs to the set $S$.

\section{Examples.}

- One can easily check that a closed interval $[a, b] \stackrel{\text { def }}{=}\{x: a \leq x \leq b\}$ is a closed set.

- On the other hand, an open interval $(a, b) \stackrel{\text { def }}{=}\{x: a<x<b\}$ is not a closed set: a sequence of elements $s_{n}=a+2^{-n}$ belongs to the open interval, but its limit $a$ does not.

What is known. It is known (see, e.g., [1]) that any function $v(S)$ that assigns, to each bounded closed set $S$, a numerical value, and which satisfies the conditions (1) and (3) for all possible sets $S$ and $S_{i}$, has the form

$$
v(S)=\alpha \cdot \sup S+(1-\alpha) \cdot \inf S,
$$

for some value $\alpha \in[0,1]$.

Comment. In [1], the results about closed-set-valued uncertainty are extended to the more general case of fuzzy uncertainty with continuous membership functions, since for these functions, $\alpha$-cuts are closed sets. We hope that our new results about general set-valued uncertainty - can be extended to fuzzy sets for which the membership function can be discontinuous. 


\section{Extending the Known Result to General Bounded Sets}

Why do we need to go beyond closed sets. Suppose that we are trying to sell something that is worth $d$ dollars, but by law, one need to have a license to sell it. So, the only way to sell is to hire someone with a license who will sell it for us. Of course, we need to pay something to that licensed person, as a result what we get is smaller than $d$. We can imagine situations in which there is a competition between licensed sellers, and we will be able to make this intermediary's cost as little as possible - but we can never make it 0 . Thus, we may be able to get gains close to $d$, but never exactly $d$.

Similarly, we can encounter situations in which licensed sellers are rare, so they can charge as much as possible - all the way to the cost $d$. They may charge $90 \%$ of the value $d$, but they cannot charge $100 \%$ - in this case, there is no sense for us to sell at all. Thus, our gain can be close to 0 , but never exactly equal to 0 .

In this case, the set of possible gains is the open interval $(0, d)-$ and we have already mentioned that an open interval is not a closed set.

Let us formulate the problem in precise terms. Now that we have motivated the need to consider generic - not necessarily closed - bounded sets, let us describe the problem of selecting the corresponding price function $v(S)$ in precise terms.

Definition 1. By a price function, we mean a function $v(S)$ that assigns, to each bounded set $S$, a number $v(S)$ in such a way that the following two conditions are satisfied:

- for every set $S$, we have inf $S \leq v(S) \leq \sup S$ (monotonicity), and

- for every two sets $S_{1}$ and $S_{2}$, we have $v\left(S_{1}+S_{2}\right)=v\left(S_{1}\right)+v\left(S_{2}\right)$ (additivity).

\section{Proposition 1.}

- Every price function has the form $v(S)=\alpha \cdot \sup S+(1-\alpha) \cdot \inf S$ for some value $\alpha \in[0,1]$.

- Vice versa, for each $\alpha \in[0,1]$, the mapping $v(S)=\alpha \cdot \sup S+(1-\alpha) \cdot \inf S$ is a price function.

\section{Proof.}

$1^{\circ}$. Due the above-cited known result, the price function $v(S)$, when limited to closed sets, it equal to $v(S)=\alpha \cdot \sup S+(1-\alpha) \cdot \inf S$ for some value $\alpha \in[0,1]$.

$2^{\circ}$. Let us now consider the simplest case of non-closed sets that we have described earlier: open intervals $(a, b)$ and - similar examples - half-open intervals $(a, b]$ and $[a, b)$. One can check that for each $a<b$, we have

$$
\begin{aligned}
& (a, b)+(a, b)=(2 a, 2 b), \\
& (a, b)+[a, b]=(2 a, 2 b),
\end{aligned}
$$




$$
\begin{aligned}
& (a, b)+(a, b]=(2 a, 2 b), \\
& (a, b)+[a, b)=(2 a, 2 b) .
\end{aligned}
$$

$2.1^{\circ}$. Indeed, let us prove (5).

- If $x \in(a, b)$ and $y \in(a, b)$, then $a<x<b$ and $a<y<b$. By adding these two inequalities, we conclude that $2 a<x+y<2 b$, i.e., $x+y \in(2 a, 2 b)$.

- Vice versa, let $z \in(2 a, 2 b)$. Then, from $2 a<z<2 b$, we conclude that $a<$ $\frac{z}{2}<b$, thus $z$ can be represented as the sum of two values $z=\frac{z}{2}+\frac{z}{2}$ from the interval $(a, b)$.

$2.2^{\circ}-2.4^{\circ}$. Similarly, we can prove equalities (6)-(8).

$2.5^{\circ}$. Due to additivity, from (5) and (6), we conclude that

$$
v((a, b))+v((a, b))=v((2 a, 2 b))
$$

and

$$
v((a, b))+v([a, b])=v((2 a, 2 b)) .
$$

Thus, we have

$$
v((a, b))+v((a, b))=v((a, b))+v([a, b])
$$

and hence, $v((a, b))=v([a, b])$.

$2.6^{\circ}-2.7^{\circ}$. Similarly:

- from (7) and (6), we conclude that $v((a, b])=v([a, b])$, and

- from (8) and (6), we conclude that $v([a, b))=v([a, b])$.

$2.8^{\circ}$. According to Part 1 of this proof, we have $v([a, b])=\alpha \cdot b+(1-\alpha) \cdot a$, thus we conclude that

$$
v((a, b))=v((a, b])=v([a, b))=v([a, b])=\alpha \cdot b+(1-\alpha) \cdot a .
$$

So, for open and half-open intervals, we have the desired property

$$
\begin{gathered}
v((a, b))=v((a, b])=v([a, b))=v([a, b])= \\
\alpha \cdot \sup (a, b)+(1-\alpha) \cdot \inf (a, b) .
\end{gathered}
$$

$3^{\circ}$. Let us now consider a generic set $S$. We want to prove that the price of this set if equal to the desired convex combination of the value $a \stackrel{\text { def }}{=} \inf S$ and $b \stackrel{\text { def }}{=} \sup S$. Our proof will depend on whether these values inf $S$ and $\sup S$ belong to the set $S$ or not. Thus, we will consider four possible cases:

- the case when inf $S \in S$ and $\sup S \in S$; 
- the case when inf $S \in S$ and $\sup S \notin S$;

- the case when inf $S \notin S$ and $\sup S \in S$;

- the case when inf $S \notin S$ and $\sup S \notin S$.

Let us consider these four cases one by one.

$3.1^{\circ}$. Let us first consider the case when $a \in S$ and $b \in S$. Let us prove that in this case, we have

$$
S+[a, b]=[2 a, 2 b]
$$

i.e., that:

- every element of the set $S+[a, b]$ belongs to the set $[2 a, 2 b]$, and

- vice versa, every element of the set $[2 a, 2 b]$ belongs to the set $S+[a, b]$.

3.1.1 ${ }^{\circ}$. Indeed, by definition of infimum and supremum, for every $x \in S$, we have $a \leq x \leq b$. When $y \in[a, b]$, we also have $a \leq y \leq b$. Adding these inequalities, we get $2 a \leq x+y \leq 2 b$, i.e., $x+y \in[2 a, 2 b]$.

3.1.2 ${ }^{\circ}$. Vice versa, let $z \in[2 a, 2 b]$.

- If $2 a \leq z \leq a+b$, then $a \leq z-a \leq b$, so $z-a \in[a, b]$ and thus, we can represent $z$ as the sum $a+(z-a)$, where $a \in S$ and $z-a \in[a, b]$.

- Similarly, if $a+b \leq z \leq 2 b$, then $a \leq z-b \leq b$, and thus, we can represent $z$ as the sum $b+(z-b)$, where $b \in S$ and $z-b \in[a, b]$.

In both case, $z \in S+[a, b]$.

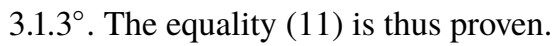

By using additivity, we conclude that $v(S)+v([a, b])=v([2 a, 2 b])$ hence $v(S)=v([2 a, 2 b])-v([a, b])$. Substituting the expression (10) for the prices of the intervals, we get the desired equality $v(S)=\alpha \cdot b+(1-\alpha) \cdot a$.

$3.2^{\circ}$. Let us now consider the case when $a \in S$ and $b \notin S$. Let us show that in this case, we have

$$
S+[a, b]=[2 a, 2 b) .
$$

3.2.1 $1^{\circ}$ Indeed, by definition of infimum and supremum, for every $x \in S$, we have $a \leq x \leq b$. Since the supremum $b$ is not attained, we have $a \leq x<b$. When $y \in[a, b]$, we have $a \leq \leq b$. Adding these inequalities, we get $2 a \leq x+y<2 b$, i.e., $x+y \in[2 a, 2 b)$.

$3.2 .2^{\circ}$. Vice versa, let $z \in[2 a, 2 b)$.

- If $2 a \leq z \leq a+b$, then $a \leq z-a \leq b$, so $z-a \in[a, b]$ and thus, we can represent $z$ as the sum $a+(z-a)$, where $a \in S$ and $z-a \in[a, b]$.

- If $a+b \leq z<2 b$, then $a \leq z-b<b$. Since $b$ is the supremum of the set $S$, by definition of the supremum, there exists a point $z^{\prime} \in S$ for which $a \leq z-b \leq z^{\prime} \leq b$. Let us show that the difference $z-z^{\prime}$ belongs to the interval $[a, b]$. Indeed, from $z-b \leq z^{\prime}$, we conclude that $z-z^{\prime} \leq b$. From the fact that 
$a+b \leq z$ and $z^{\prime} \leq b$ thus $-b \leq-z^{\prime}$, we conclude that $(a+b)+(-b) \leq z+\left(-z^{\prime}\right)$, i.e., that $a \leq z-z^{\prime}$. So, the value $z$ can be represented as $z^{\prime}+\left(z-z^{\prime}\right)$, where $z^{\prime} \in S$ and $z-z^{\prime} \in[a, b]$.

$3.2 .3^{\circ}$. The equality (12) is thus proven. By using additivity, we conclude that $v(S)+v([a, b])=v([2 a, 2 b))$ hence $v(S)=v([2 a, 2 b))-v([a, b])$. Substituting the expression (10) for the prices of the intervals, we get the desired equality $v(S)=$ $\alpha \cdot b+(1-\alpha) \cdot a$.

$3.3^{\circ}-3.4^{\circ}$. Similar arguments show that:

- when $a \notin S$ and $b \in S$, then $S+[a, b]=(2 a, 2 b]$ and thus, $v(S)=\alpha \cdot b+(1-\alpha) \cdot a$; and

- when $a \notin S$ and $b \notin S$, then $S+[a, b]=(2 a, 2 b)$ and thus, too

$$
v(S)=\alpha \cdot b+(1-\alpha) \cdot a .
$$

The proposition is proven.

\section{What If We Do Not Require Additivity?}

Formulation of the problem. What if we do no require additivity - e.g., if we are talking about preferences in general, not necessarily monetary preferences. In this case, as we will show, we can still reduce a general set to the case of an interval.

First reasonable requirement. If $a<b$, then clearly $b$ is better. For the case of uncertainty, when we have two sets $A$ and $B$, and:

- each alternative $a$ from the set $A$ is smaller then or equal to some alternative $b$ from the set $B$, and

- each alternative $b$ from the set $B$ is larger than or equal to some alternative $a$ from the set $A$,

then it is reasonable to conclude that $B$ is better or of the same quality as $A$; we will denote it by $A \leq B$.

If $A \leq B$ and $B \leq A$, we will denote it by $A \equiv B$. Let us describe it in precise terms.

Definition 2. Let $\leq$ be a reflexive $(A \leq A)$ and transitive relation on the class of all bounded subsets of the real line. We say that this relation is monotonic if for every two sets $A$ and $B$ for which the following two properties are satisfied:

- for every $a \in A$, there exists $a b \in B$ for which $a \leq b$, and

- for every $b \in B$, there exists an $a \in A$ for which $a \leq b$,

we have $A \leq B$.

Proposition 2. For every monotonic relation, each bounded set is equivalent to an interval: 
- if $\inf S \in S$ and $\sup S \in S$, then $S \equiv[\inf S$, $\sup S]$;

- if $\inf S \in S$ and $\sup S \notin S$, then $S \equiv[\inf S$, sup $S$ );

- if inf $S \notin S$ and $\sup S \in S$, then $S \equiv(\inf S$, sup $S]$;

- if $\inf S \notin S$ and $\sup S \notin S$, then $S \equiv(\inf S$, sup $S)$.

Proof. We will consider the four cases one by one. Let us denote $s_{-} \stackrel{\text { def }}{=}$ inf $S$ and $s_{+} \stackrel{\text { def }}{=} \sup S$.

$1^{\circ}$. Let us first consider the case when $s_{-} \in S$ and $s_{+} \in S$. To prove that in this case, we have $S \equiv\left[s_{-}, s_{+}\right]$, we need to prove that $S \leq\left[s_{-}, s_{+}\right]$and that $\left[s_{-}, s_{+}\right] \leq S$. Let us use Definition 2 to prove these two inequalities.

$1.1^{\circ}$. Let us first prove that $S \leq\left[s_{-}, s_{+}\right]$; indeed:

- For each $s \in S$, by definition of supremum, we have $s \leq s_{+}$; since $s_{+} \in\left[s_{-}, s_{+}\right]$, the first property from Definition 2 is satisfied.

- Similarly, for each $s \in\left[s_{-}, s_{+}\right]$, we have $s_{-} \leq s$. Since $s_{-} \in S$, the second property is also satisfied.

Thus, indeed, $S \leq\left[s_{-}, s_{+}\right]$.

$1.2^{\circ}$. Let us now prove that $\left[s_{-}, s_{+}\right] \leq S$; indeed:

- For each $s \in\left[s_{-}, s_{+}\right]$, we have $s \leq s_{+}$; since $s_{+} \in S$, the first property from Definition 2 is satisfied.

- Similarly, for each $s \in S$, we have $s_{-} \leq s$. Since $s_{-} \in\left[s_{-}, s_{+}\right]$, the second property is also satisfied.

Thus, indeed, $\left[s_{-}, s_{+}\right] \leq S$.

$1.3^{\circ}$. So, indeed, $S \equiv\left[s_{-}, s_{+}\right]$.

$2^{\circ}$. Let us now consider the case when $s_{-} \in S$ and $s_{+} \notin S$. To prove that in this case, we have $S \equiv\left[s_{-}, s_{+}\right)$, we need to prove that $S \leq\left[s_{-}, s_{+}\right)$and that $\left[s_{-}, s_{+}\right) \leq S$. Let us use Definition 2 to prove these two inequalities.

$2.1^{\circ}$. Let us first prove that $S \leq\left[s_{-}, s_{+}\right)$; indeed:

- For each $s \in S$, by definition of supremum, we have $s \leq s_{+}$; since the supremum is not attained, we have $s<s_{+}$; thus, we have $s \leq s$ for $s \in\left[s_{-}, s+\right)$. So, the first property from Definition 2 is satisfied.

- Let $s \in\left[s_{-}, s_{+}\right)$, then $s_{-} \leq s$. Since $s_{-} \in S$, the second property is also satisfied.

Thus, indeed, $S \leq\left[s_{-}, s_{+}\right)$.

$2.2^{\circ}$. Let us now prove that $\left[s_{-}, s_{+}\right) \leq S$; indeed:

- For each $s \in\left[s_{-}, s_{+}\right)$, we have $s<s_{+}$. Since $s_{+}$is the supremum of the set $S-$ i.e., the least upper bound, and the value $s$ is smaller that $s_{+}$, this means that $s$ is not the upper bound for the set $S$. In other words, this means that there exists a value $s^{\prime} \in S$ which is not smaller than or equal to $s-$ i.e., which is larger than $s$ : $s<s^{\prime}$. So, the first property from Definition 2 is satisfied. 
- Now, for each $s \in S$, we have $s_{-} \leq s$. Since $s_{-} \in\left[s_{-}, s_{+}\right]$, the second property is also satisfied.

Thus, indeed, $\left[s_{-}, s_{+}\right) \leq S$.

$2.3^{\circ}$. So, indeed, $S \equiv\left[s_{-}, s_{+}\right)$.

$3^{\circ}-4^{\circ}$. Similarly, we can prove the equivalence for two other cases. The proposition is proven.

Relation between open, closed, and half-open intervals. To analyze the relation between open, closed, and half-open intervals, it makes sense to take into account that, as we mentioned earlier, we do not see the difference between $\varepsilon$-closed values if the $\varepsilon>0$ is sufficiently small.

Definition 3. We say that a reflexive and transitive relation $\leq$ between bounded subsets of the real line is realistic if for every two sets $A$ and $B$, for which the following two conditions hold:

- for every $a \in A$ and for every $\varepsilon>0$, there exists a value $b \in B$ which is $\varepsilon$-close to $a$; and

- for every $b \in B$ and for every $\varepsilon>0$, there exists a value $a \in A$ which is $\varepsilon$-close to $b$,

we have $A \equiv B$.

In this case, it is easy to prove the following result:

Proposition 3. For each realistic relation, for all $a<b$, we have

$$
[a, b] \equiv(a, b) \equiv(a, b] \equiv[a, b) .
$$

Thus, intervals of different type are equivalent to each other - provided that their endpoints coincide. For monotonic relations, in view of Proposition 2 , every bounded set $S$ is equivalent to an interval - thus, if this relation is also realistic, we always have $S \equiv[\inf S, \sup S]$.

\section{Acknowledgments}

This work was supported in part by the National Science Foundation grants 1623190 (A Model of Change for Preparing a New Generation for Professional Practice in Computer Science), and HRD-1834620 and HRD-2034030 (CAHSI Includes). It was also supported by the program of the development of the Scientific-Educational Mathematical Center of Volga Federal District No. 075-02-2020-1478. 


\section{References}

1. J. Lorkowski, R. Aliev, and V. Kreinovich, "Towards Decision Making under Interval, SetValued, Fuzzy, and Z-Number Uncertainty: A Fair Price Approach”, Proceedings of the IEEE

World Congress on Computational Intelligence WCCI'2014, Beijing, China, July 6-11, 2014. 